\title{
Hospital User Research using New Media Arts
}

\author{
Cecily Morrison \\ Computer Laboratory, \\ University of Cambridge \\ JJ Thompson Ave, \\ Cambridge, UK, CB3 0FD \\ Cecily.Morrison@cl.cam.ac.uk
}

\author{
Alan F. Blackwell \\ Computer Laboratory, \\ University of Cambridge \\ JJ Thompson Ave, \\ Cambridge, UK, CB3 0FD \\ Alan.Blackwell@cl.cam.ac.uk
}

\begin{abstract}
This paper presents a comparative analysis of group interaction around two display types, shared and individual, using a 'new media' arts application as a way to explore the physical technology setup for an intensive care unit in a hospital. We propose this method for laboratory settings when the research questions derive from socially complex environments, but realworld interventions are not possible. While users solve an 'interaction problem' that is posed through the 'new media' arts application for their own expressive purposes, researchers can analyse and collate the results to understand the solution space. We present a study with the bodyPaint application to address a design issue that we discovered when assessing the merits of an electronic patient record system.
\end{abstract}

\section{Author Keywords}

Intensive Care, Mobile Devices, New Media Arts

\section{ACM Classification Keywords}

H.1.2. User/Machine Systems - Human Factors; H5.2. User Interfaces - Evaluation/methodology;

\section{INTRODUCTION}

Health-related technologies have recently become a topical area in the field of Human-Computer Interaction (HCI). The majority of this work however, consists of applications to help individuals monitor their own health. Despite a world-wide push by governments to improve healthcare and lower costs by using technology in hospitals, such as the UK's £10bn National Program for Information Technology [13] and Sierra Leone's initiative to build an integrated health information system [1], there is still relatively little related research in academic HCI settings that focuses on technology (as opposed to studies of paper in medical settings, e.g. [5]). We suggest that this dearth comes from the difficulties of doing research in the critical environment of the hospital. Even if ethical approval can be obtained, doctors can be hesitant to introduce innovative technology that might disrupt patient care.

We recently experienced this problem while working in an intensive care unit. Having carried out an observational study of ward round interaction using paper and electronic patient

(c) The Author 2009.

Published by the British Computer Society records [12], we noted a conflict between how the multidisciplinary team of 8-10 practitioners would have liked to gather around the patient record to interact productively and what was possible with the 19" monitor on which the electronic patient record (EPR) was displayed. Consequently, we wanted to assess whether mobile devices would more effectively support ward round team interaction than static displays. We proposed a 2 week study using tablet PCs, but unfortunately, despite the research-orientation of the unit, interventional research (as opposed to observation) was deemed infeasible in such an environment.

Unable to explore the above question in the hospital environment, we looked for ways to do so in the laboratory. However, as [14] notes, results from experiments done in a laboratory do not translate easily to socially complex settings, because most of the social context is lost. It is this research tension between work in the laboratory and external validity that we engage with in this paper.

Following information on the research problem in the 'Background' section, the 'Research Methodology' section reviews a number of problems associated with designing laboratory studies, pointing out particular issues that the study presented here is meant to address. We then propose a 'new media' arts application as an element in an adjusted design cycle as one way of doing a laboratory-based study that is relevant to a particular real-world scenario. We move from the theoretical to the practical by presenting a study that assesses how two display types affect the negotiation of interaction utilizing the application bodyPaint.

\section{BACKGROUND}

\subsection{The Research Problem}

The research problem explored in this paper is inspired by a previous ethnographic study comparing ward round interaction around paper versus electronic patient records in the intensive care unit (ICU) of a specialized cardiothoracic hospital [12]. The patient record is the central focus of the ward round, which brings together 8-10 staff from a range of disciplines and domains of expertise each day to assess jointly the patient's condition and required future care. In order to provide good medical care, the team must collaborate, or to be more specific, carefully negotiate the interaction, so that all appropriate information and expertise are shared, but no time is wasted. The aim of the previous study was to understand how the negotiation of the interaction differed between the two records.

Realizing that the change from a tangible presentation of information in the paper record as opposed to the non-tangible electronic record was likely to affect non-verbal behaviour [8], and that non-verbal behaviour is a significant part of co-located group interaction [11], we focused the ethnographic study on 

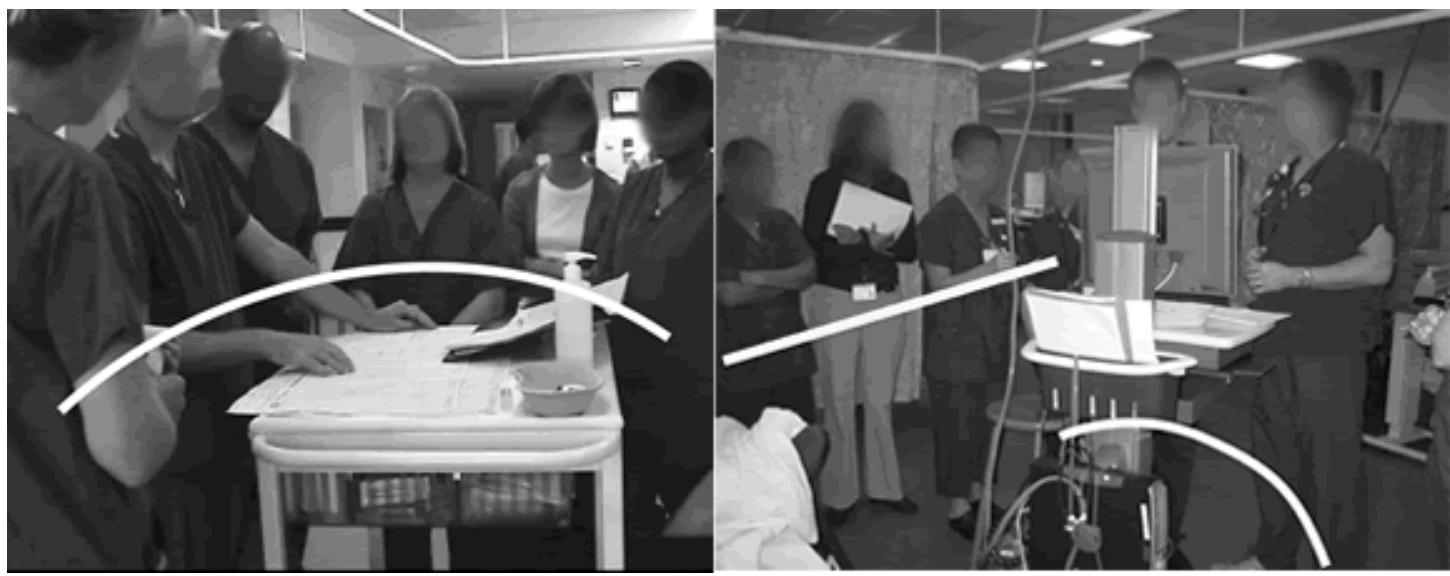

Figure 1: Ward Round teams using a paper patient record (left) and an electronic patient record (right).

observing the physical nature of the interaction, i.e. the use of bodies and objects. We drew upon the work of Kendon and his theory of the F-formation system [9] to do this, which describes how groups form, adjust and disband their formations in order to negotiate entrance into, or progression of, the interaction. We observed, in agreement with Kendon, that certain formations allowed team members to use non-verbal behaviours to negotiate interaction, and others did not. We also noted that the physical setup of the technology used, could influence formation as in Figure 1, which contrasts the formation of a ward round team using a paper patient record (left) and an electronic patient record (right).

There is tension between formation changes that occur as a means of interaction negotiation and ones that occur because of physical setup of the technology. It is this conflict and how teams solve it that this study aims to probe.

In the hospital context of the example pictured in Figure 1, the physical setup of the EPR, a 19" display at the end of the patient bed, restricted the formation of the team and consequently the participants' ability to feel part of the team and to utilize non-verbal behaviours in negotiating the interaction. One way to defuse this tension between differing formation needs might be to use mobile devices that allow people to configure themselves as necessary. However, it is possible that using mobile devices would create new and equally problematic difficulties. Posture, for example, affects people's ability to interact, as does access to information - two things likely to change with mobile devices. To begin this investigation on the effect of display type on the negotiation of interaction, we look at what people do at the two extremes, posing the following research question:

How does a team negotiate interaction differently when using a large display as opposed to individual, small displays?

\section{STUDY METHODOLOGY}

Studies in HCI frequently draw upon experimental methods to evaluate interface design decisions in the laboratory [2]. Experimental approaches have worked well for applications in which a single person uses a desktop machine. More recently, experimental methods have been applied to technologies used by groups [4]. In the former case, there are many studies in which social context that is relevant to the application use does not differ dramatically between in-situ and laboratory environments. This is not true for the latter case in which the social context may dramatically alter the way that groups interact and therefore use technology during interaction. The following section examines issues of social context in the choice of participants, task, and development of measurements for studies of co-present groups using technology.

\subsection{Participants}

Laboratory studies assessing group interaction, with few exceptions e.g. [16], do not state the composition of groups. Perhaps the most important characteristic of group composition to group interaction is whether the group has previously done similar types of interaction. Some studies note whether participants know each other or know each other well, mentioning that this is a factor likely to affect collaboration, but do not distinguish between types of knowing. I would argue that previous experience in doing the kind of task in the study together is more likely to influence the study then familiarity gained from knowing someone. Working as a team for example, suggests different relationships with people than being friends. An important study design choice in the study presented below is that the participants have an established way of negotiating interaction as a group.

\subsection{Task}

Motivation is an important element of a task in studies that focus on group interaction. Social interaction depends on shared experience of previous social interaction, in which emotional reaction to certain situations and people often develop. Those emotions will affect how people react in a situation. People are more likely to be willing to overcome difficulties in interaction, for example, when someone's life is at stake, as in a hospital, than when the outcome is unimportant. As this is an acknowledged issue, some researchers offer monetary rewards to increase motivation, such as [4]. Whether this is appropriate motivation depends on the person and his or her culture. Anticipated financial rewards might help maintain motivation over repetitive tasks, but does not account for motivation being an emotional response (e.g. anger or strong sense of equality) which is likely to be a factor in collaborative interaction in a real-world setting.

There are examples in the HCI literature of successful ways to motivate collaboration, particularly the emotional side of interaction. Games tend to have motivation built into them with a surrounding culture of play and expectation. They have the further advantage of having been refined over time and are thus more likely to have rules that encourage interaction. [19] translated the Towers of Hanoi puzzle into a game for two people, for example, to study collaboration between the sighted 


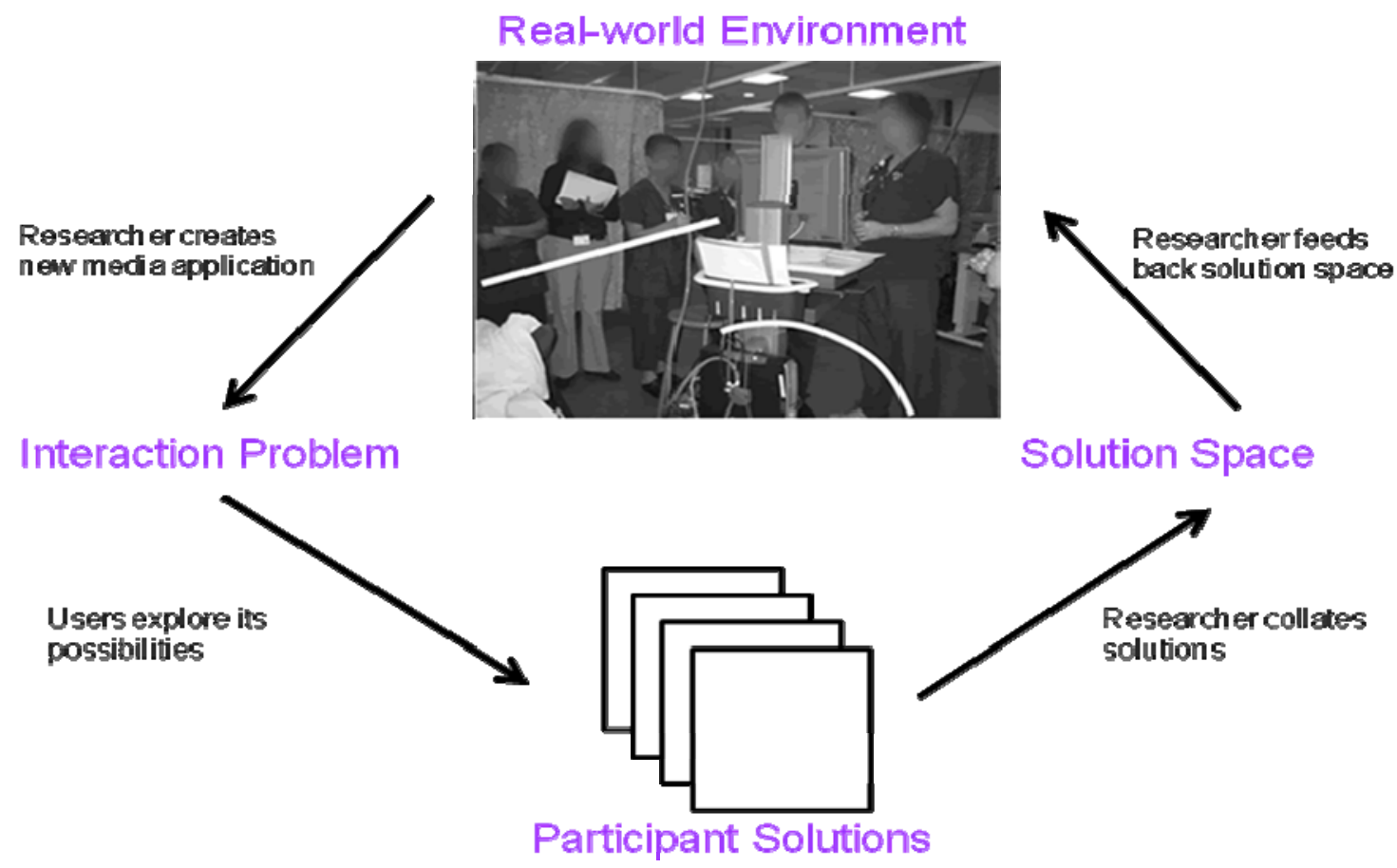

Figure 2: Using 'new media' arts to get from an interaction problem to a solution space

and the blind. Established games, however, may not be available for all types of interaction.

An alternative is creative work, such as [16]. In creative interaction motivation comes from a sense of ownership and desire to achieve ones artistic goals [7]. As an additional benefit, creative work is more likely to encourage exploration and thus be generative as well. Consequently, in the study presented below, we have chosen to use a creative game as a way to increase motivation to negotiate interaction in such a way that would be reasonably comparable to the real-world environment of a hospital.

\subsection{Measures}

Measures, unlike participants and task, do not influence the social context of the group per se, but rather how it is recorded. Collaboration, the most common type of group interaction investigated in HCI settings, is a word with a well understood tacit meaning, but is difficult to define. Studies done in the field skirt this issue by defining collaboration in terms of the context. For example, one of the design goals of [15] was to relieve the socially awkward moments between travel agent and customer. The authors specified the difficulties in the ethnographic work and described how the new technology addressed these in the evaluation. [3], taking a different approach, had a model of collaborative group interaction built into the technology and tested its suitability by deploying the technology and assessing its usage. In both cases, what was meant by collaboration was defined before the design of the evaluation, either through the needs of the participants or through the technology itself.

It is more difficult to assess collaboration in the laboratory, in which the measures rather than the context define what collaborative group interaction means. In some cases group interaction is quantifiable, such as the number of conflicts resolved in a study of conflict negotiation. In many HCI settings, the researcher is interested in the quality of the interaction and whether the technology improves it. When investigating quality, measures become second order, counting some behaviour that is considered indicative of better quality but is not directly correlated, such as equal participation or speech. The difficult lies in balancing between what is easily measured in the laboratory and what needs to be measured to provide results useful for developing technology for a realworld scenario.

This problem is exacerbating in quantitative laboratory studies which require a base line against which to measure change. In some areas of research, such as remote collaboration tools, the base line is face-to face interaction. When the goal of research is to improve upon the existing base line, new ways of generating and analysing data need to be considered.

One alternative to second order measures and quantitative base lines is theory-structured qualitative research. [19], for example, which investigates communication possibilities of a blind and a sighted person when using the same interface, structures their analysis around elements of Conversational Analysis. They focus on how the interface supports turn-taking and speech acts, which are known to be ubiquitous elements of interaction. The analysis investigates when and how these take place and the specific role of the technology or application in facilitating the communication. The use of theory provides useful context to the analysis but is not burdened with the difficulties of how it might be quantified and what the baseline should be.

Theory-structured qualitative methods in the laboratory do not, however, solve the problem of how to create an explorative environment to generate possibilities beyond the base line. Along with a complementary way of thinking about groups as the unit of analysis and task motivation, the design of the study presented in this paper focuses on the problem of generating possibilities that go beyond the baseline. It is an exploration of doing design in a very different way. Before introducing the role of this study in the design process, I will present the impetus to work in this manner which comes from 'new media' arts. 

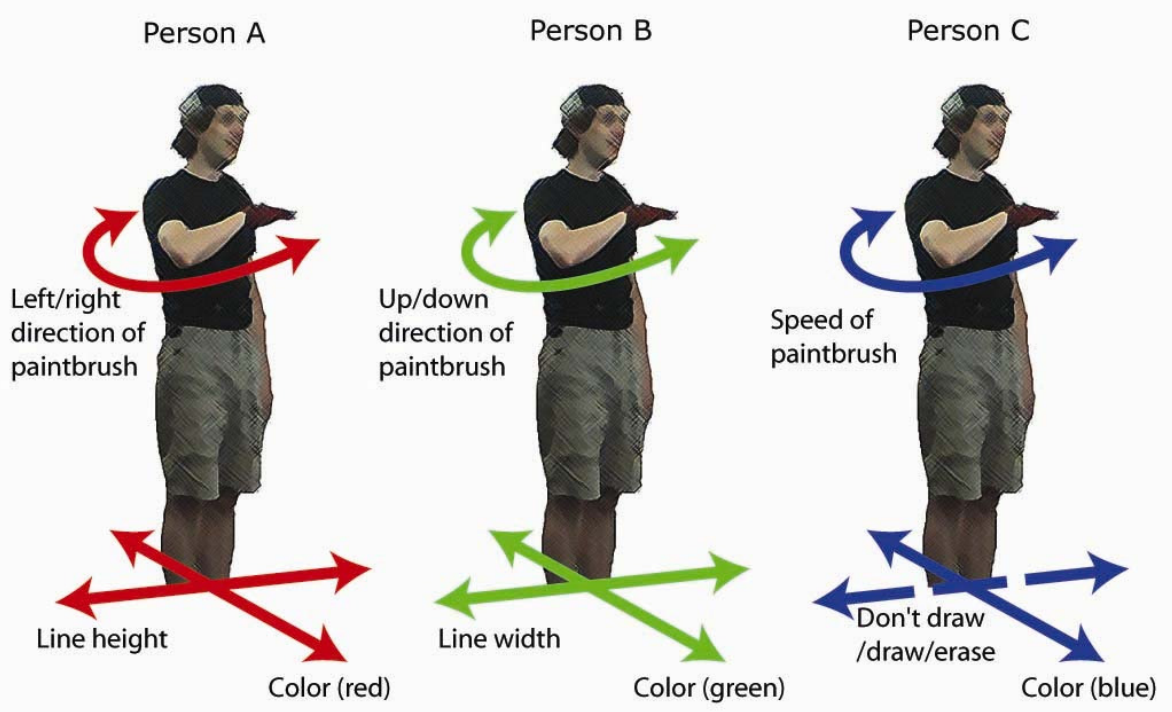

Figure 3:bodyPaint Controls

\subsection{A 'New media' Arts Approach}

Suchman [18] argues in Human-Machine Reconfigurations that 'new media' arts is fertile ground for exploring such complex $\mathrm{HCI}$ issues as the one central to this thesis. Her discussions lead the reader away from the prevalent computer science occupation with determining and debating the agency of the 'smart' machine towards a depiction of agency as a construction made by humans using machines. She suggests that while Artificial Intelligence as a field is occupied with the former debate, many 'new media' artists, those who work with digital technologies as creative tools, are on the forefront of exploring how humans give machines agency. As artists, their approach is not one of reasoned possibility, but one meant to provoke an experience in viewers so that they explore the boundaries for themselves.

The design of the bodyPaint application follows a similar approach by motivating people to explore an 'interaction problem' by using a 'new media' arts application. In pursuit of their own personal artistic goals, people generate a large number of possible ways of interacting. The result is a solution space of possible interactions for the researcher to analyse. Knowing the boundaries of what is likely and possible, the researcher can then advise those in the real-world scenario as to the starting points for developing their interaction and the technology that supports it. This process is pictured in Figure 2. The use of the application as the task provides the motivation, but most importantly, generates ideas that move beyond the baseline approach of most laboratory studies.

The process into which the laboratory study is embedded also diminishes some of the measurement issues discussed above. Second order measures are not necessary because the aim of the result is not to determine the best way, but the possible ways of interacting and what causes them to break down. Moreover, the need to provide all social context in order to achieve external validity is minimised. Only the social context relevant to the interaction under study needs to be facilitated in order to generate solutions as the actual choice and evaluation of a solution happens in the real-world situation. In the case of the study presented below, the application needs to be designed to produce negotiation of interaction around different displays rather than capture the dynamics of the medical team.

\subsubsection{Application Design}

The design of the application, as described below, maximises negotiation of interaction and does not attend to either the types of tasks done with the EPR or the particular people doing them. This stands in contrast to a more usual approach of simulating an activity in the laboratory. It is easy to assume that since the simulation 'looks' like a ward round, then the results are automatically 'correct' and applicable to the original environment. This is not the case. The simulation does not necessarily provide the motivation of the original environment. The medical practitioners do not have any reason to solve conflicts between themselves as the patient actor is not in danger. It is however, important to focus on one aspect of interaction, in this case the negotiation of interaction, in order to avoid some of the problems of general laboratory studies.

The aim of the study is to produce as many and varied solutions as possible. Asking a ward round team to be participants and do what they usually do is unlikely to be generative. Most teams have established ways of working and may not be keen to experiment. Using other participants provides a fresh perspective on how one might go about solving this particular interaction problem. It is possible that the solution space is 'a little off' because of the differences in people and task. Small differences however, can be provocative when the 'results' are fed back to the medical team. For example, real-time updating is present in the study but not in the real environment. If realtime updating facilitates group interaction, then that suggests trying an interactive application.

\section{STUDY DESIGN}

\subsection{The Artistic Experience (The Task)}

\subsection{1 bodyPaint Application}

bodyPaint is an interactive multi-person paint program in which three users control a single on-screen brush with their movements. It utilizes a Vicon motion capture system to track the position of participants' torsos and hands in threedimensional space. Horizontal hand motion of the three participants controls the direction vector: the $\mathrm{x}$-coordinate, the $y$-coordinate and the speed respectively. Moving around in the $2 \times 3$ meter grid controls the colour, line width, and drawing mode as indicated more precisely in Figure 3. The novel control mechanism, split between three people, used to manipulate the 


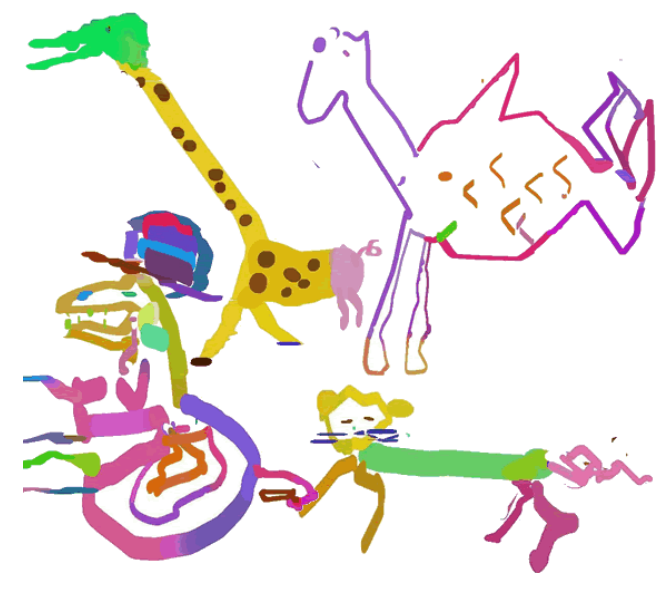

Figure 4: Animals drawn with the bodyPaint application

system in order to achieve the group goal, is intended to encourage group exploration of movement and interaction.

\subsubsection{The Challenge}

Teams are introduced to the application with written and verbal directions. Afterwards, they are asked to draw a square with sides of differing widths and a multi-coloured, unattached circle. They are free to ask questions while figuring out how the application works. The teams are then given the creative challenge of drawing their 'dream pet,' depicting a creature that includes recognizable features from at least three animals. Example creations are shown in Figure 4. This challenge is designed with the dual purpose of giving the participants some structure to get them started and of accentuating the negotiation of interaction.

Teams first need to negotiate which aspects of each animal to include, where to draw them, and how to shape them. For example, if they decide to draw the head of a giraffe, the body of a fish and the tail of a rooster, not only does the team need to negotiate a shared understanding of what that combined creature looks like (i.e. size, position, shape) but to adapt that understanding as the picture develops and team members opportunistically take advantage of the unintended, depending on their positions and the state of the drawing. The constant need for negotiation gives researchers the opportunity to watch the negotiation process develop over time with various devices.

\subsection{The Teams (The Participants)}

\subsubsection{Group Selection}

We invited four teams of three people each to use the system by asking one individual to bring two people that $\mathrm{s} / \mathrm{he}$ had known for over a year and had collaborated with in some way (e.g. organized an event, worked closely on a publication or project). Fitting with the research model proposed in this paper, we wanted a diverse set of teams in order to increase the number of solutions to the 'interaction problem.' The more solutions there are, the more likely an improvement on the base line measure will be found. As shown in Figure 5, the teams ranged from a design team to a family. We limited the number of teams to four in order to carry-out detailed video-analysis of their respective solutions.

\subsection{The Data Capture and Analysis (The Measurements)}

\subsubsection{Method and Theory}

The goal of the data analysis, as put forward in the research model, is to describe the solution space of the interaction problem that we posed to the teams. We use video-based interaction analysis to generate a rich description of each solution. To structure the analysis we drew upon F-formation Systems, a theoretical means of describing how people negotiate interaction by adjusting their formation [9]. This theory looks at the relationships between people in a group and the consequences that has for interaction.

\subsubsection{Modes}

In line with the research question posed above, "How does a team negotiate interaction differently when using a large display as opposed to individual, small displays?" -- We asked the teams to solve the 'interaction problem' in two modes. The first mode required the use of individual small screens strapped to the left arm (Figure 6b), the second -- a wall projected display 1.5 meters in front of the capture space (Figure 6a). We focused on the two extremes of display type in order to gather solutions that contrasted as much as possible, without significantly disrupting the creative experience. We did this by switching displays after twenty minutes, giving each team up to forty minutes to create its drawing. The teams did not find this switch disturbing, but used the break to discuss what they had done and what they would like to do. This format was chosen because initial use of the application suggested that a minimum of thirty minutes was needed to complete a drawing but that more than twenty minutes was too long for individuals to hold the small display units without tiring.

\subsubsection{Data Analysis}

We collated and organized these individual solutions into a description of the solution space by focusing on three areas of analysis: formation, learning, and preference.

Formation -- Our original query was whether there was a conflict between using formation to negotiate interaction and the formation possibilities of the physical set-up of a given

\begin{tabular}{|l|l|}
\hline Group 1 & Postgraduate research team \\
\hline Group 2 & Family \\
\hline Group 3 & Design team for education software \\
\hline Group 4 & Trio of Musicians \\
\hline
\end{tabular}

Figure 5: Group Participants

piece of technology. We investigated this comparing how Kendon expects groups to act and how we observed them when using the bodyPaint application.

Learning -- With two different 'interaction problems' to solve, it is likely that a team's second solution will incorporate elements of the first. Although this could bias the results in a standard laboratory study, we avoided this problem by making learning one of the main themes of the analysis. For this reason, both the learning period and the main task were videoed and analysed.

Preferences -- A third area of interest was whether preference for one display type over another reflected differences in team members' abilities to negotiate the interaction. We gained data on preferences through a fifteen minute discussion following the use of the bodyPaint application. 


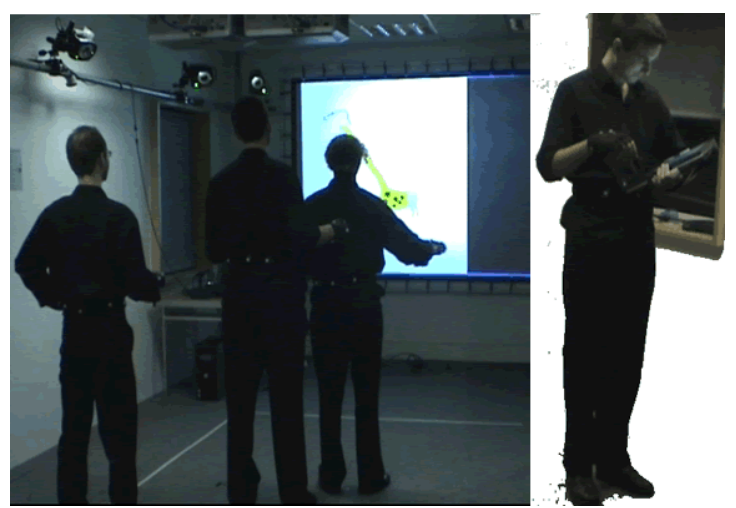

Figure 6: large, wall-projected display (left); individual, small display (right)

The first of these areas of analysis queries whether mobile devices should be recommended for trial in the hospital setting. The latter two investigate how severe problems caused by an unhelpful physical setup are, a likely situation in a hospital with budget constraints. The breadth of this enquiry provides helpful suggestions to clinical leaders who need to make decisions, without providing a single 'right' answer that is unlikely to be externally valid.

\section{ANALYSIS}

\subsection{Formation}

This study was developed to investigate the conflict between the use of formation to negotiate interaction and the formation possibilities imposed by the physical set-up of the technology in use. The aim of the analysis then is to tease out when adapting to the technology has a detrimental effect on the negotiation of interaction. We will begin by detailing the possibilities of interaction that a formation allows, according to Kendon. We will then see if the formations of the groups differ from that which Kendon describes, and if so, whether the interaction possibilities have been lost or shifted to another medium.

\subsubsection{Kendon}

Kendon [9] suggests that formation is a way of depicting relationships between people. One relationship is between those inside versus those outside of the group. For this reason, formations are generally circular and inward facing as depicted in Figure 7a. A related capability that derives from an inward facing formation is the creation of a shared interaction space. The break in formation when the EPR was introduced, shown in Figure $1 b$, is a good example of how a formation change caused by the technology did not define clearly who was in the group and who was not. Nor did it provide an alternative shared interaction space. The result was lack of interaction and wandering attention.

The shape of the formation demonstrates relationships between the people in the formation. In a horseshoe shaped formation, as in Figure $7 b$, some people, depending on their position, have more access to the interaction space. For example, the person at the top has greater access to the interaction space and this person's non-verbal cues are more visible to others, allowing this person to lead and coordinate. The consultant's position at the head of the horseshoe shape in front of the paper patient record, in Figure 1a, is an example of preferential access to the interaction space and ability to lead that were lost when the EPR was introduced.

\subsubsection{Teams}

Formations developed by the four teams did not differ dramatically. The teams oriented themselves towards the wall where the large display was located, as shown in Figure 8,

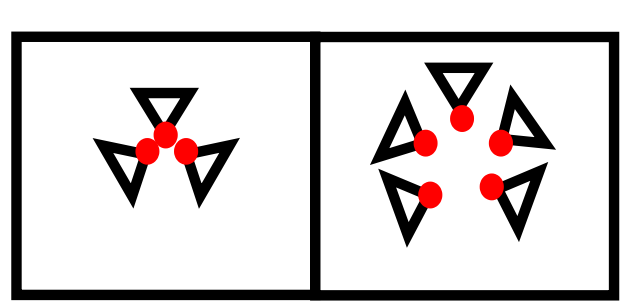

Figure 7: Kendon Formations

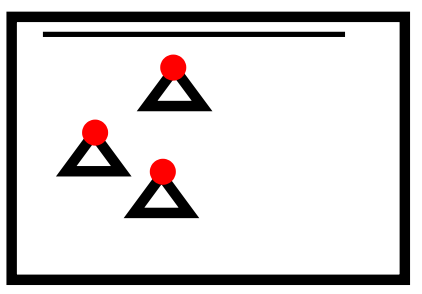

Figure 8: Typical Team Formation

regardless of the display type in use. Their spatial relationship, although partly determined by the teams' drawing needs, tended to be circular in nature. When teams needed to be in a more linear configuration to draw, one often bent down or turned slightly, maintaining a sense of circular dimension, rather than linearity. I would characterize the formation as circular, but not inward facing.

The predominant team formation differed from Kendon's examples. Consequently, we looked for alternative ways used by the teams to satisfy interactional needs that an inward-facing circular formation would have provided, notably (1) creating a shared focus; (2) establishing leadership or facilitating coordination; and (3) using non-verbal behaviours to negotiate the interaction on other levels.

\subsection{Discussion}

\subsubsection{Shared Focus}

The formations of the teams made it impossible for the shared focus to be the enclosed interaction space as in the Kendon description. One possibility is that the happenings in the entire room made up the shared interaction space, something that might be tested if multiple groups were in the space simultaneously. Observation indicates however, that the focal point of the interaction space was the display(s). All teams spent the majority of their time with their members' eyes 'glued' to the display. A number of teams explicitly stated that the large display was a means for having a shared focus.

\subsubsection{Interaction negotiation}

The forward-facing orientation necessary when using the large display would make it difficult for team members to access the non-verbal actions that Kendon suggests groups use to negotiate interaction. Although a circular formation might have been difficult to achieve in relation to the large display, the small displays allowed people to orient towards each other. Nonetheless, they chose not to orient towards each other. Indeed, they stated a preference for the large display. They reasoned that the position of their heads, straight ahead rather than focused down towards the small display, made it easier to monitor their team-mates and their team-mates' movements.

Although non-verbal actions seemed unnecessary in coordinating activity when the teams were drawing, they reverted to using them and the associated formations when discussing their drawings during the technical break or at the end of the session. Non-verbal behaviours were also employed if there was a breakdown in communication. The most common 
strategy was first to instruct each other verbally if the drawing was not going according to plan and then strengthen the verbal communication with a turn of the head, followed by a reorientation of the body, and as a last resort, use of a gesture indicating what to do. This suggests that non-verbal behaviours (in this context) are most important when there is a misunderstanding or conflict but are not necessary all of the time. The amount of non-verbal behaviour also seemed to depend on the personality and expressive habits of each person.

\subsubsection{Coordination}

Physical coordination, so that team members did not bump into each other, was not a problem. As soon as someone entered the space of another, the stationary person compensated, as one would expect. An additional means of coordination was the mental model of the grid, the $2 \times 3$ meter space in which the team members moved to control the paint brush. Even when the teams used individual displays, they oriented towards the large display, also the top of the grid, in contrast to the expectation that they would face inward. A number of people commented that this facing helped them know in which direction they should move and to predict where others might be.

Coordination of the activity was another matter. Across teams there was a general consensus that the large display was preferred because it made monitoring the actions of others easier. Teams used different strategies for coordinating the task: two teams utilized a previous shared experience and two had to create rules as they went along. The design team for example, used very little verbal communication, relying heavily on preestablished interaction roles: the idea generator, the approver, and the helper. The idea generator proposed what was to happen, the approver said 'yes' or 'no,' and then they carried the action out. The team of research colleagues exploited their shared knowledge of Greek mythology and how creatures are made from different animals in order to describe what needed to happen.

The other two teams had more difficulty coordinating and took longer to settle on a strategy. One team took nearly five minutes to draw the first line of their pet as they could not communicate what part of it they were drawing or what it looked like. Eventually team members relinquished individual control and allowed a leader to emerge who barked out orders to the group. Sarcastic comments about the leader in the discussion suggest this was not an optimal solution. The second team also had a leader who directed everyone's action. However, this team agreed upon what they were drawing through a series of short consultations during which they formed a Kendon-style formation around the screen (both large and small) and used gestures to define the shape of the image.

\subsection{Conclusions}

An inward-facing circular formation provides a transaction space and a means to non-verbally negotiate the interaction. The teams in this study did not employ such a formation, but demonstrated a number of other ways of achieving the same level of interaction. Shared-focus was provided by the display; the real-time update encouraged constant monitoring. Shared experience or concepts were also employed in a virtual 'sketch pad' for considering and coordinating ideas before they were drawn. Despite these substitutes, nobody was comfortable when $\mathrm{s} /$ he could not monitor, at least peripherally, the other people on the team. This was seen both in the desire for the large screen and the careful rearranging of heights when a linear formation was needed to draw.
Although the inward-facing formation that facilitates nonverbal behaviours to negotiate the interaction was abandoned, non-verbal behaviours remained essential to the interaction at certain times. Although the most common usage was to resolve conflicts or crisis, they were also important at other times. For example, the family created an inward-facing formation to facilitate non-verbal behaviour when planning, or re-planning, their drawing. The music trio, unable to solve the interaction problem because their practiced non-verbal behaviours did not serve them in this context, had to resort to an autocratic leader. These results suggest that there are alternatives other than an inward-facing circle for productive interaction around technology, but that the alternatives should be closely scrutinized because not all of them are optimal. Moreover, the conclusions demonstrate that a shared perspective, e.g. a shared display, is not enough to support social interaction, despite this being a commonly proposed design rationale (e.g. [10]).

\subsubsection{Learning}

Watching the learning process was a particular goal of this study. Both the 'learning period' with the technology as well as the creative task itself were videoed and subject to analysis. The learning period demonstrated a number of formations tried and discarded, indicating the relative importance of various formation factors. Second, the main task period revealed whether the form-factor was prominent enough to change established ways of interacting.

Two groups learned the application with the large display and two with the small one. The two that learned it with the large display faced toward it in both modes, not even considering an alternative formation with the small display. Those teams that learned the application with the small display began drawing in an inward-facing formation. This dissolved within the first five minutes as team members began to move along the grid. The inward-facing formation was again tried when the small display was used after the large display, but disappeared even more quickly. This immediately suggested that the inward-facing formation was not necessary for coordination. It also indicated that the physical set-up of the technology does not seem to dictate entirely the choices of how participants organize themselves.

\subsubsection{Preferences}

Another important issue was how preference changed observable behaviour, which I explored in a short focus group following the activity. I asked team members to sit around a one meter square table covered with paper. They were asked three questions and encouraged to write or draw before answering in turn; they were told that they could freely react to what others said. The questions were:

1. Did you prefer the large screen or the small one? Why?

\section{How did your group make decisions about what to do} next?

3. If you could have displays of any size, anywhere in the room, where would you put them?

In each of the four groups, two team members preferred the large display and one the small display. In all four cases, the person who preferred the small display used a lot of non-verbal actions -- gesticulation, engaged posture, and turning to face the person spoken to. The small displays facilitated the use of nonverbal action. Nonetheless, the display type did not change either the teams' coordination strategies or the formations. This 
implies that preferences do not affect people's ability to participate in the team.

The last two questions did not lead to any helpful information. The teams had difficulty describing their decision-making process, and their thoughts as to how things might have been different were not particularly imaginative or revealing. At this point the participants were very tired, though, and they did not seem to feel like answering questions.

\subsubsection{Application to Hospital Environment}

Several of the insights acquired as a result of the study are applicable to further exploration by the ward round team in the hospital. First, fostering a shared perspective could be useful in circumventing some possible issues introduced by new technology and should be considered as part of the development of a system. This would not have to relate to an application specifically, but could be linked to the information structure or a social protocol. For example, the intensive care unit in our ethnographic study had a particular order of relaying information in the ward round. It should also be noted that when the physical setup of the technology makes the negotiation of interaction with non-verbal behaviours impractical, and there is no shared perspective, there is the danger that some team members will be unable to participate.

Second, the deployment of individual small displays for each team member is probably not a useful direction for further exploration as the resulting posture of use makes it difficult to monitor the interaction. The incorporation of small displays in other technology setups might be a more productive avenue in order to gain the tangible advantage of paper to work in parallel [17]. Third, individual preference for a display type does not affect how a team coordinates. It seems that teams can adapt to a variety of technology form-factors if given the opportunity. Although their ability to do this should not be the default, it should be taken into account when considering tradeoffs.

\section{DISCUSSION}

We have presented arguments for why a 'new media' arts application is an alternative way of exploring design issues. We proposed that by posing an 'interaction problem' to a team in the form of a 'new media' artistic experience, the team, pursuing their own expressive purposes, would provide a solution. The solutions of many teams could then be explored with video analysis and collated into a description of a solution space. This solution space can then be presented back to those in the original research scenario to support their exploration and decision making processes. We outlined a study that we carried out with the bodyPaint application to explore possible conflicts in the use of formation as a way of negotiating interaction, and the formation possibilities provided by a piece of technology with a given physical setup.

The analysis suggested that: (1) a shared perspective can be an important means of supporting interaction when non-verbal actions cannot be used and thus should be considered in the design process; (2) individual small displays for everyone are not a design option that should be pursued when designing technology set-ups for semi-mobile, co-located groups, but that their incorporation into a mixed technology set-up might be more useful; and (3) the display type did not change the coordination strategy in the cases here, but that teams adapted to get the job done.

We look forward to observing the ward round interaction with paper support and with one person having a mobile device in the future.

\section{ACKNOWLEDGMENTS}

We would like to acknowledge the team of people that we worked with at Papworth Hospital as well as funding contributions from Boeing Inc.

\section{REFERENCES}

[1] E-Health in Sierra Lionne. http://www.friendspartners.org/utsumi/Global_University/Global\%20University\% 20System/Africa/Sierra\%20Leone/Fund\%20Raising 07-2106/Executive\%20Summary\%20of\%20GUS_Sierra\%20Leone $\%$ 20Project/GUS.Sierra\%20Leone\%20exec.\%20summary\%206.2 9.06_v10\%20copy.pdf

[2] Barkhuus, L, Rode, J. (2007). From Mice to Men: 24 years of evaluation in CHI. Proc. of CHI 2007.

[3] Benford, S., Rowland, D., Flintham, M., Drozd, A., Hull, R., Reid, J., et al. (2005). Life on the Edge: supporting collaboration in location-based experiences. Proc. of CHI 2005.

[4] Birnholtz, J., Grossman, T., Mak, C., \& Balakrishnan, R. (2007). An exploratory study of input configuration and group process in a negotiation task using a large display. Proc of CHI 2007.

[5] Cabitza, F., Sarini, M., Simone, C., \& Telaro, M. (2005). When once is not enough: the role of redundancy in a hospital ward setting. Proc. of Group 2005.

[6] DiMicco, J.M, Pandolfo, A and Bender, W. (2004). Influencing Group Participation with a Shared Display. Proc. of CSCW 2004

[7] Gauntlett, D. (2007). Creative Explorations: new approaches to identities and audiences. Routledge.

[8] Heath, C. (1986). Body-movement and Speech in Medical Interaction. Cambridge University Press.

[9] Kendon, A. (1990). Conducting Interaction: patterns of behaviour in focused encounters. Cambridge University Press.

[10] Koyabashi, K. Narita, A. Hirano, A. Kase, I, Tsuchida, S, Omi, T, Kakizaki, T, Hosokawa, T. (2006). Collaborative simulation interfaces for planning disaster measures. Proc. Of CHI 2006.

[11] Koschmann, T. LeBaron, C. (2003). Reconsidering Common Ground: examinging Clark's Contribution Theory in the OR. Proc. of ECSCW 2003.

[12] Morrison, C. Jones, M. Blackwell, A. Vulysteke, A. (2008). Electronic Patient Record use during ward rounds: a qualitative study of interaction between medical staff. Critical Care Forum. http://ccforum.com/content/12/6/R148

[13] National Program for Information Technology www.connectingforhealth.co.uk

[14] Robson, C. (2002) Real-World Research. 2nd Edition. Blackwell Publishing.

[15] Rodden, T., Rogers, Y., Halloran, Y., \& Taylor, I. (2003). Designing novel interaction workspaces to support face to face consulations. Proc. of CHI 2003.

[16] Rogers, Y, Hazlewood, W, Blevis, E, Lim, Y. (2004). Finger Talk: collaborative decision-making using talk and fingertip interaction around a tabletop display. Proc. of CHI 2004. 
[17] Sellen, A. Harper, R. (2001). The Myth of the Paperless Office. MIT Press.

[18] Suchman, L. (2007). Human-Machine Reconfigurations. Cambridge University Press.
[19] Winberg, Bowers, J. (2004). Assembling the Senses: towards the design of cooperative interfaces for visuallyimpaired users. Proc. of CSCW 2006. 